\title{
Vector Control Analysis of Aedes Mosquitoes: Past, Present and Future Athanasia Karagiannis
}

\author{
Satesh Bidaisee ${ }^{1 *}$ and Siriluk Pichainarongk ${ }^{2}$ \\ ${ }^{1}$ St. George's University, Grenada, West Indies \\ ${ }^{2}$ Kasetsart Univerity, Thailand \\ *Corresponding author: Satesh Bidaisee, Siriluk Pichainarongk, Kasetsart Univerity, Thailand
}

\begin{tabular}{|c|c|}
\hline ARTICLE INFO & ABSTRACT \\
\hline Received: 䳆 February 22, 2021 & $\begin{array}{l}\text { The Aedes aegypti and Aedes albopictus mosquitoes are the most prevalent invasive } \\
\text { species contributing to the worldwide spread of endemic and zoonotic diseases, such }\end{array}$ \\
\hline Published: March 01, 2021 & \\
\hline $\begin{array}{l}\text { Citation: Satesh Bidaisee, Siriluk } \\
\text { Pichainarongk. Vector Control Analysis of } \\
\text { Aedes Mosquitoes: Past, Present and Future } \\
\text { Athanasia Karagiannis. Biomed J Sci \& Tech } \\
\text { Res 34(2)-2021. BJSTR. MS.ID.005525. }\end{array}$ & $\begin{array}{l}\text { competence for multiple arboviruses and pathogens, reinforces their prominence as a } \\
\text { global public health threat. With increasing climate change and expansion in human travel } \\
\text { and trade, the threat of Aedes mosquitoes spreading to areas of the world where limited } \\
\text { resources and insufficient vector control programs exist is troublesome. Reviewing } \\
\text { current technological advancements, integrated vector management, and global } \\
\text { engagement is important to improve environmental, chemical, biological, and genetic }\end{array}$ \\
\hline $\begin{array}{l}\text { Keywords: Vector Control; Aedes } \\
\text { Mosquitoes; ae. Aegypti; ae. Albopictus }\end{array}$ & $\begin{array}{l}\text { vector control methods used in disease prevention. The present study is a narrative } \\
\text { review of the past, present, and future vector control strategies and perspectives of Aedes }\end{array}$ \\
\hline $\begin{array}{l}\text { Abbreviations: HAT: Human African } \\
\text { Trypanosomiasis; IRS: Indoor Residual } \\
\text { Spraying; ITN: Insecticide-Treated Bed Net; } \\
\text { F: Lymphatic Filariasis }\end{array}$ & $\begin{array}{l}\text { mosquitoes to support and propose new public health initiatives that prevent and control } \\
\text { mosquito disease transmissions globally. It is hypothesized that along with the existing } \\
\text { vector control practices in place, there is a need for continued integrated vector and case } \\
\text { management, sustainable government and community cooperation, and further research } \\
\text { on novel vector control methods to globally mitigate the spread of Aedes-borne viruses. }\end{array}$ \\
\hline
\end{tabular}

\section{Introduction}

Mosquitoes are among the most prolific invasive species contributing to the worldwide spread of endemic and zoonotic diseases [1]. The two most prevalent species are Aedes aegypti and Aedes albopictus (European Centre for Disease Prevention and Control [ECDC], 2017). They can transmit a variety of re-emerging arboviruses (arthropod-borne) that usually have no vaccine or disease-specific treatment, such as chikungunya, Zika, Dengue, yellow fever, and West Nile [2]. Due to incessant climate change and the expansion of human travel and trade, Ae. aegypti, originally from Africa, and Ae. albopictus, originally from Asia, have now spread to all continents, except Antarctica [3]. Both species are daytime biters and feed on humans and animals, increasing the risk of human bites, since it is more difficult to take protective measures during the day than at night, when bed nets are more effective [4]. Additionally, they can feed on multiple individuals within a short period of time, spreading disease more rapidly. Their eggs are incredibly resistant, having the ability to survive during the winter, out of water, and to tolerate a wide range of temperatures. Furthermore, they can be transported in large numbers over long distances, inhabiting tires, water storage containers, and plants. Originally from tropical and forest natural habitats, they have successfully adapted to suburban and urban environments, increasing their expansion capability as more countries become urbanized [5].

A range of biological, chemical, and environmental vector control and surveillance methods have been implemented globally to prevent Aedes expansions; however, most developing areas lack the resources and organized mosquito control to effectively respond to new arrivals and infections [6]. Thus, it is important to review current vector control measures and anticipate areas with potential establishment of Aedes mosquitoes, in order to develop successful public health campaigns against future disease outbreaks. This narrative review analyzes past and present mosquito control programs and existing challenges to help inform future practices on how to mitigate this emerging global public health threat [7]. It is 
hypothesized that, along with the existing vector control practices in place, there is a need for continued integrated vector management, sustainable government and community cooperation, and further research on novel vector control methods to globally reduce the spread of Aedes-borne viruses [8].

\section{Materials and Methods}

\section{Search Methods}

The online databases used to perform relevant literature searches were Google Scholar, Academic Search Complete, Vet Science, and MEDLINE with Full Text. Boolean/Phrase search modes were utilized to maximize search results, by combining related terms, such as "mosquito control" and "vector control." Keywords used under advanced searches included: Aedes aegypti and Aedes albopictus mosquitoes, specific biological, chemical, and environmental vector control and surveillance methods, public health interventions, programs, preventions, vector-borne diseases, projections, and past, present, and future strategies and perspectives. The literature search was done from August 2017 through June 2020. In considering article selections, the titles and abstracts were assessed. Twenty articles were selected and analyzed to assess public health initiatives that prevent and control Ae. aegypti and Ae. albopictus mosquito disease transmissions.

\section{Inclusion Criteria}

Articles included were in the English language only and focused on Ae. aegypti and Ae. albopictus mosquitoes specifically and their global vector control methods and initiatives. Search results were limited to include full text, scholarly peer reviewed articles, and published literature from 2010 to 2020.

\section{Exclusion Criteria}

Articles excluded were in languages other than English and that involved other Aedes species, such as Ae. australis, Ae. cinereus, and Ae. polynesiensis.

\section{Analysis}

Aedes vector Control: Vector control remains the main existing method to protect against most Aedes transmitted diseases due to limited or no commercially available vaccines and drug treatments [9]. Currently, there are only two licensed vaccines against Aedes-borne diseases: a widely used yellow fever 17D vaccine which produces rapid, lifelong immunity, and a recently licensed Dengue vaccine (Dengvaxia) that is used in 19 countries but carries a potential risk of severe disease in Dengue-naïve individuals, making it a safety concern for global administration [10]. Other vaccine candidates for Dengue, Zika, and chikungunya are currently in different clinical trial phases [11]. Therefore, vector control methods that largely depend on removing or reducing human-vector contact are used globally to restrict Aedesviral transmissions [12]. Broadly, Aedes control measures can be separated into environmental, biological, and chemical-based tools [13].

Environmental Vector Control Methods: Before the introduction of chemical insecticides, such as DDT (dichlorodiphenyl-trichloroethane) in 1940, vector control was mainly limited to environmental management, which focused on disrupting local breeding sites and manipulating vector behavior and ecology [14]. Types of environmental vector control include house screens, aquatic habitat drainage, vegetation clearance, water container coverage, hygienic measures, waste management, protective clothing, and various other agricultural and housing improvements [15]. Looking back at the history of vector control practices, a form of environmental management was always implemented since past generations successfully connected fevers to the proximity of surface waters, like swamps and marshes [16]. There are reports of ancient Greeks, Romans, and Egyptians using drainage schemes, bed nets, and curtains as mechanical vector control measures to prevent mosquito bites [17]. Additionally, during the late 1700s, yellow fever was controlled in the US by pumping bilge water out of ships and cleaning sewers [18]. Although labor intensive, these environmental vector control methods proved largely successful in controlling yellow fever epidemics in the Americas during the early 1900s [19]. The disease was almost eliminated, but due to reduced political support and vector surveillance following its success, the yellow fever vector, Ae. aegypti, was able to reestablish itself throughout the Americas during the late 1900s. This outcome demonstrated that maintaining government support and investment in Aedes vector control methods was paramount in preventing the resurgence of arboviruses [20].

Chemical Vector Control Methods: Following the discovery of DDT in 1940, contact-based insecticides became the leading vector control method against mosquitoes, since they were less labor intensive and rapidly effective [21]. Insecticides derive from four main classes: pyrethroids, organophosphates, organochlorines, and carbamates. Types of chemical vector control include residual insecticide spraying, insecticide-treated bed nets (ITN) and curtains, and larvicides. Today, most vector control tools are characterized into chemical and non-chemical methods, targeting either the immature larval or adult stage of vectors (Figure 1). For example, immature vectors are killed by microbial/chemical larvicides and predator species and/or reduced by aquatic habitat removal or modification, such as surface water drainage or coverage. Mature vectors are primarily killed by Indoor Residual Spraying (IRS), space spraying, and insecticide treatment and/or reduced by limiting human bites, using house screenings, bed nets, topical repellants, and removal trappings. 


\begin{tabular}{|c|c|c|c|}
\hline \multirow[t]{10}{*}{ Chemical } & Immature & Chemical larvicides & $\begin{array}{l}\text { Contact pesticides affecting insect nervous system (e.g., temephos) or endocrine system (insect } \\
\text { growth regulators, e.g. pyriproxyfen) }\end{array}$ \\
\hline & \multirow[t]{9}{*}{ Adult } & ITNs & $\begin{array}{l}\text { Pyrethroid-treated ITNs or combination ITNs (e.g. pyrethroid plus synergist piperonyl } \\
\text { butoxide) for malaria, L.F, and leishmaniasis control }\end{array}$ \\
\hline & & $\begin{array}{l}\text { Insecticide-treated materials for personal } \\
\text { protection }\end{array}$ & Insecticide-treated clothing for workers and mobile populations \\
\hline & & IRS & $\begin{array}{l}\text { Spraying of residual insecticides (typically either pyrethroids, carbamates, or } \\
\text { organophosphates) indoors for malaria and Aedes-borne disease control }\end{array}$ \\
\hline & & Space spraying & $\begin{array}{l}\text { Aircraft, vehicle or hand-held space spraying for dengue epidemic and other Aedes-borne } \\
\text { disease control }\end{array}$ \\
\hline & & Insecticidal treatment of habitat & Focal, perifocal, ground, or aerial insecticide spraying \\
\hline & & Insecticide-treated cattle & Pour-on or spot-on pyrethroids for control of tsetse \\
\hline & & Insecticide-treated traps and targets & $\begin{array}{l}\text { Targets for control of HAT and insecticide-treated adulticidal oviposition traps for Aedes- } \\
\text { borne diseases }\end{array}$ \\
\hline & & Topical repellent & $\begin{array}{l}\text { Chemicals (e.g, N,N-diethyl-meta-toluamide [DEET], picaridin) applied to the skin to reduce } \\
\text { vector biting }\end{array}$ \\
\hline & & Spatial repellent & Transfluthrin/metafluthrin passive emanators or coils \\
\hline \multirow[t]{7}{*}{ Nonchemical } & \multirow[t]{5}{*}{ Immature } & Microbial larvicides & Bacillus thuringiensis var, israelensis, $B$. sphaericus \\
\hline & & Predator species & Predatory fish or invertebrates \\
\hline & & $\begin{array}{l}\text { Habitat modification, i.e, a permanent } \\
\text { change of land and/or water }\end{array}$ & $\begin{array}{l}\text { Drainage of surface water, land reclamation and filling, and coverage of large water storage } \\
\text { containers (or complete coverage of water surfaces) with a material that is impenetrable to } \\
\text { mosquitoes, such as expanded polystyrene beads }\end{array}$ \\
\hline & & $\begin{array}{l}\text { Habitat manipulation, i.e., a recurrent } \\
\text { activity }\end{array}$ & $\begin{array}{l}\text { Water-level manipulation, exposing habitats to the sun (depending on the ecology of the } \\
\text { vector), flushing of streams, drain clearance, and source reduction, including rubbish disposal } \\
\text { and regular emptying and cleaning of domestic containers (e.g., flowerpots, animal drinking } \\
\text { water troughs) }\end{array}$ \\
\hline & & Regulatory measures & Removal of man-made aquatic habitats and appropriate waste disposal \\
\hline & \multirow[t]{2}{*}{ Adult } & House improvement and screening & Closing eaves, door and window screening \\
\hline & & Removal trapping & $\begin{array}{l}\text { Solar-powered mosquito trapping system for malaria control and sticky adulticidal oviposition } \\
\text { traps for Aedes-borne diseases }\end{array}$ \\
\hline
\end{tabular}

Figure 1: Examples of chemical vs. non-chemical vector control methods for immature vs. adult vectors.

Unfortunately, insecticide resistance has already become widespread in Ae. aegypti and is emerging in Ae. albopictus, threatening the efficiency and availability of insecticide-based vector control for many human arboviruses. Due to the limited number of approved insecticides, it is difficult to prevent resistance using only rotation cycles. Insecticide resistance is a natural selection process, influenced by environmental, biological, and genetic factors, that gives insects an inherited ability to survive a toxicant dose that would normally be lethal to a susceptible population of the same species under the same conditions (Figure 2). Resistance can also result from rare de novo mutations, but is more common in species showing high fecundity, short generation time, dominant resistant alleles, and existing genetic variation. Therefore, the implementation of Insecticide Resistance Management (IRM) strategies in vector control is essential to maintain the efficiency of existing insecticides. These strategies work by reducing the overall selection pressure and fitness of resistant insect populations by rotating insecticides, mixing dissimilar insecticides, and mosaic spraying.

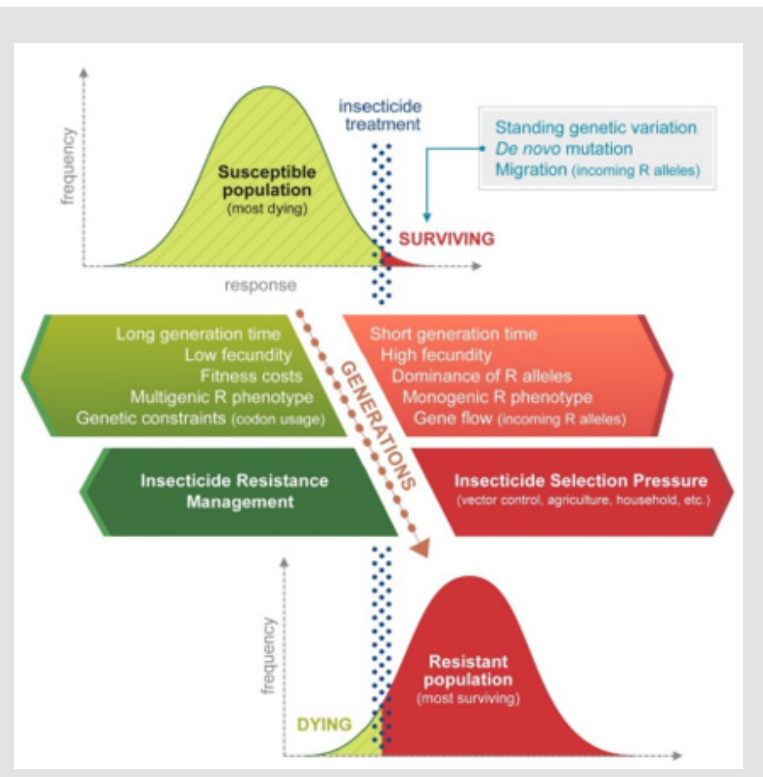

Figure 2: Factors influencing insecticide resistance selection in insect populations. The red dotted line corresponds to the population's evolutionary response to an insecticide dose across many generations of insecticide selection. The proportion of individuals (resistant population) surviving the insecticide dose and the factors that favor resistance selection are shown in red, while the factors that impair resistance selection, which includes Insecticide Resistance Management (IRM) are shown in green. 
This ensures that there is no cross-resistance or similar modes of action. Resistance management is most successful when it is not used as the sole strategy but integrated with other non-insecticide control methods, along with monitoring tools, such as bioassays that track insecticide resistance. Moreover, residual insecticides, like DDT, have social concerns of causing environmental harm and human health damage. As a result, using non-insecticide approaches when available are preferred. Other novel chemicalbased measures that aim to reduce insecticide resistance include the use of ingested insecticides and semiochemicals in odor-based traps. Types of ingested insecticides include Attractive Toxic Sugar Baits (ATSB), which kill Ae. albopictus by targeting their nectarfeeding behavior to deliver the insecticide, and Dried Attractive Bait Stations (DABS), which kill Ae. aegypti in field and semifield experiments by using visual attractive cues and boric acid as the lethal ingested agent. Since mosquitoes use a complex of semiochemicals to search for blood-hosts, sugar meals, mates, and oviposition sites, correctly identifying and selecting these cues for use in various odor-based traps can be highly efficient in controlling Aedes mosquito populations.

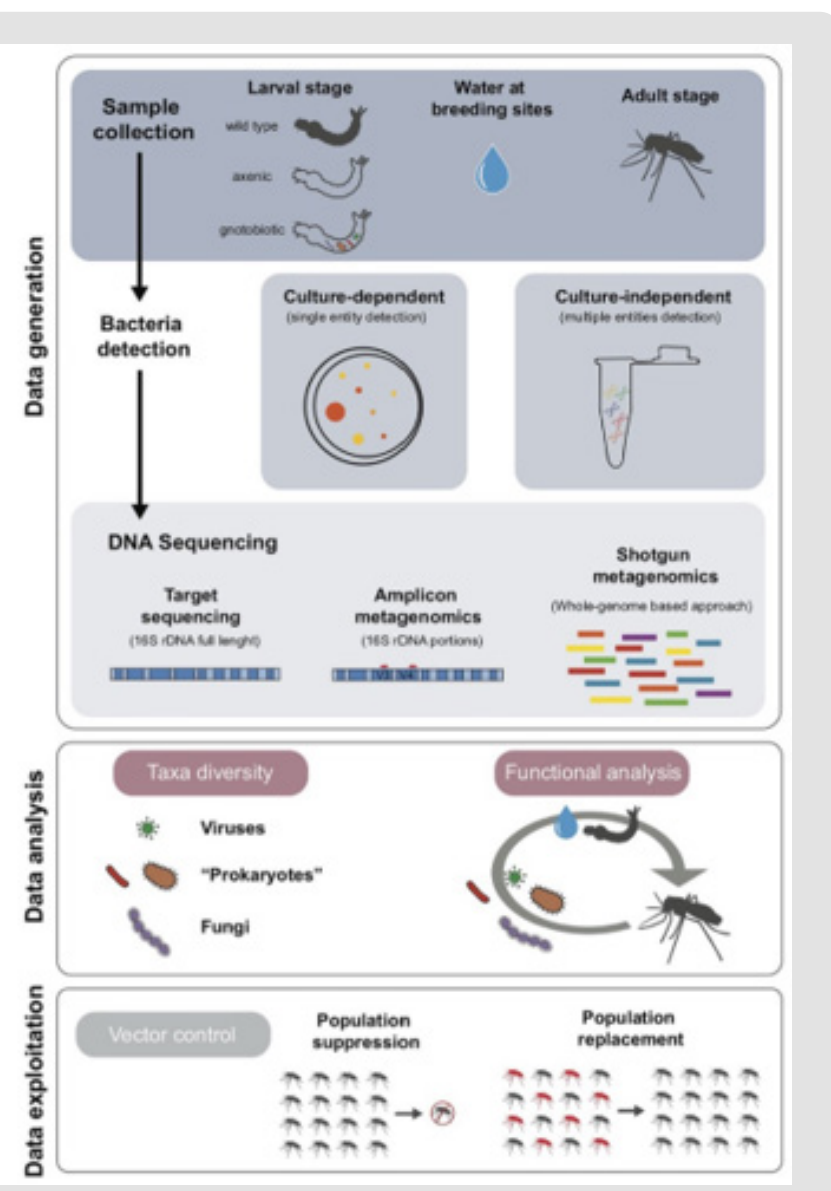

Figure 3: Stages of Aedes microbiota studies: data generation, analysis, and exploitation.

Bacterial Vector Control Methods: Since 2001, the study of gut microbiota or host-associated microbes in mosquitoes has provided new environmentally friendly vector control strategies that target Aedes mosquitoes. The study of Aedes microbiota can be organized into three main stages: data generation, analysis, and exploitation (Figure 3). The microbiota influences the physiological processes of mosquitoes, affecting their reproduction, growth, survival, and response to external stressors. Types of biological vector control methods include larvivorous fish, copepods, entomopathogenic fungi, and the bacteria, Bacillus thuringiensis israelensis, which target breeding sites and kill larval and adult mosquitoes. The bacteria genus, Wolbachia, has been successfully used to reduce Aedes mosquito transmissions of Dengue, by inducing oxidative stress and cytoplasmic incompatibility to inhibit Dengue vector competence. Moreover, genetically modifying the mosquito's natural symbionts to express anti-viral properties has been another effective strategy.

Vector competence is the ability of an arthropod (mosquito) to obtain, support replication and distribution of a pathogen, and successfully transmit it to other susceptible hosts (humans). It is influenced by the mosquito's microbiota, environmental, and genetic factors. An example of a genetic factor that can be manipulated to reduce the mosquito's vector competence is nonretroviral Integrated RNA Virus Sequences (NIRVS). By activating these immune pathways, effector genes with antiviral properties can be expressed in the mosquito, inhibiting the arboviral competency. Another form of genetic modification is known as gene drive, which incorporates favorable traits through interbreeding mosquito populations by altering or replacing genes. This method uses population replacement/modification, which reduces vector competence by replacing existing wild mosquito populations with traits that cannot transmit pathogens, and population suppression, which reduces or eliminates mosquito populations by reducing female fertility or increasing males. An example of a successful population suppressed strain of mosquito is the "flightless female" Ae. aegypti, which have a toxin-coded gene that destroys their wing muscles, preventing them from mating and searching for food and breeding sites.

Recently, gene editing in Aedes mosquitoes using the CRISPRCas9 system has become a more efficient, direct, and cheaper method of vector control. It uses Clustered Regularly Interspaced Palindromic Repeats (CRISPR), which are loci found in various bacteria and archaea, and CRISPR-associated Cas9 genes, to create site-specific mutations that inhibit incoming pathogens in mosquitoes. This new genome-engineering tool has expanded the ability to modify genomes of many different organisms, creating stable mosquito germline mutations that can improve gene drive strategies and be integrated with other existing vector control methods. Nonetheless, several challenges exist with gene editing that require consideration, such as laboratory-developed techniques being successfully applied to field releases, accidental migrations of genetically modified mosquitoes outside of intended release zones, and gene drive resistance. Unwanted mutations in gene edited mosquitoes can arise after field releases, requiring 
reversible mechanisms in gene drive strategies. The development of threshold-dependent drive systems that express disease-refractory genes in Aedes populations, in a confined and reversible manner, has become a reliable solution. This approach provides localized population control, without the threat of a wide scale spread of genetically modified mosquitoes. Additionally, the risk of accidental migrations into bordering countries can result in the repopulation of previously eliminated local mosquitoes and create political tensions with vector control campaigns. Therefore, adequate surveillance, sampling, and detection of mosquitoes near political borders needs to be implemented with gene drive strategies to avoid unwanted invasiveness. Lastly, plans to reduce gene drive resistance include targeting multiple gene sites for disruption, instead of having a single-target Cas9-based gene drive, to avoid the development of gene drive resistant alleles.

\section{Surveillance Methods}

Effective Aedes surveillance relies on the accurate and rapid identification of collected mosquito samples to guide vector control programs. A range of surveillance methods exist that assess vector abundance and distribution and the risk of human exposure to infected mosquitoes. Standard "exposure-free" methods include indirectly estimating human-vector contact rates by surveying mosquito larvae in water containers and collecting resting adults in or around houses. To better assess the risk of human exposure to arboviral infections and predict potential outbreaks, "host-seeking" trapping methods, such as BG-sentinel (BGS) and Mosquito Electrocuting Traps (MET) are used to directly measure human biting rates. These traps use attractive odor and visual cues to lure mosquitos in and kill them on contact. Other methods of Aedes surveillance include laboratory-based techniques, such as molecular and PCR-based assays, that can more accurately identify specific mosquito species in field samples and differentiate between similar species. However, these techniques require a reliable electrical supply, costly laboratory equipment, and trained personnel, which is limited in most countries with endemic Aedes arboviral infections. Ultimately, the choice of surveillance method will depend on the country's geographical and temporal distribution of infected mosquitoes, budgetary and logistical constraints, and availability of skilled personnel.

\section{Integrated Vector Control Management}

Even though a wide variety of vector control methods exist, many countries still lack the resources, funds, preparedness, and guidance to implement sustainable vector control interventions. The Integrated Aedes Management system (IAM) is a framework developed by the Worldwide Insecticide Resistance Network (WIN) that helps health authorities design appropriate vector control strategies to reduce the burden of Aedes-borne infections in their area. It incorporates 4 pillars of action (integrated entomological and epidemiological surveillance, vector control, social mobilization, and multi-sectoral collaboration), along with supporting national activities (capacity building, advocacy, research, and policies and laws), that are tailored to local Aedes viral transmission and distribution risks (Figure 4). The framework also supports the guidelines underlined by the World Health Organization Global Vector Control Response (WHO GVCR), which provide countries with evidence-based recommendations on how and when to implement practical vector control interventions.

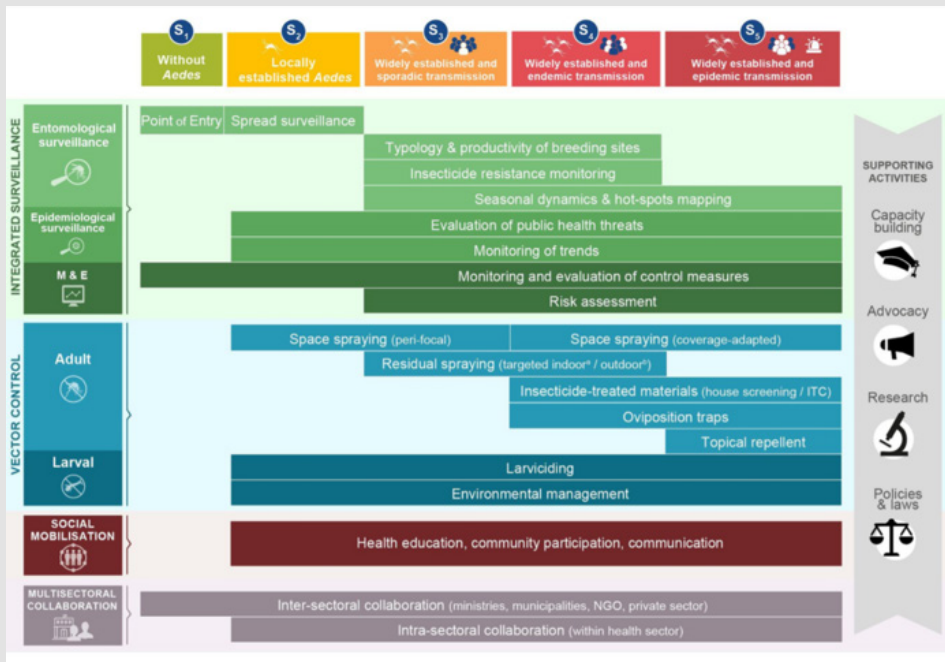

Figure 4: Integrated Aedes Management (IAM) framework that includes 4 activity pillars (integrated surveillance, vector control, social mobilization, and multisectoral collaboration) and 4 supporting activities (capacity building, advocacy, research, and policies and laws) that apply to different local Aedes transmission risk scenarios.

a) Ae. albopictus

b) Insecticide Treated Curtain (ITC); Monitoring and Evaluation (M \& E); Nongovernmental Organization (NGO). 
Vector control is most enhanced when there is community participation and collaboration among multiple partners within and outside the health sector. It is important to engage and educate the public on properly identifying, removing, and treating Aedes mosquito aquatic habitats in and around their homes, to help reduce vector populations. Moreover, integrating social marketing techniques within vector control interventions can positively influence voluntary behavior changes at the individual (downstream), community (midstream), and government/ policy (upstream) levels. Activities promoting behavioral change, through health education programs and mass media, can lead to better health outcomes, such as reducing infection rates. It is also important to consider different vector control dynamics that can influence continued community participation, such as government authority, media, socioeconomic status, social norms, collective awareness, people's attitudes, herd immunity, climate, and infrastructure. Additionally, the public health sector should work together with those involved in waste management, water, sanitation, urban planning, environment, and housing, to ensure proper management of Aedes habitats and vector control methods. Vector control management is most successful when there is intraand intersectoral collaboration that includes case management and diagnostics, vaccine delivery, maternal and child health, environmental health, veterinary and epidemiological surveillance, and other vector-borne disease programs.

\section{Conclusion}

After reviewing the various past and present vector control methods used to reduce Aedes-transmitted diseases, vector control management has proved to be a highly effective approach, when integrated with community and multi-sectoral action and collaboration. There is a need for continued investment and political support in establishing Aedes vector control as a public health priority to prevent disease resurgence. Ideally, implementing a combination of environmental, chemical, biological, and genetic vector control methods, along with constant vector surveillance, monitoring, and evaluation of these interventions, would produce the most effective results. However, in countries or areas with limited resources, funding, and capacity for highly effective vector control programs, locally adapted problem-solving approaches can be established that use successful vector control methods from the past. For example, environmental management and larval control programs can be implemented where genetically modified mosquitoes and other more costly biological control methods are inaccessible.

Ae. aegypti and Ae. albopictus mosquitoes remain a global public health threat, due to their rapid evolutionary adaption to different breeding sites, feeding behavior, and climate variation, along with their competence for multiple arboviruses and pathogens. Aedesborne diseases, such as Dengue, Zika, yellow fever, and chikungunya have resurged or expanded globally over the past 50 years. Dengue viral infections have been on the rise, with epidemics recurring every 3 to 5 years in the Americas and infecting about 390 million humans per year. Yellow fever has re-emerged in the Americas and Africa, mostly due to the migration of unvaccinated people into endemic areas. Zika and chikungunya outbreaks have spread worldwide since the 2000s, linked with deforestation, increased temperatures, and rainy seasons. Thus, establishing long-term Aedes vector control programs, combined with improvements in water storage, sanitation, waste disposal, human health resources, funding, community engagement, and political will, are necessary to globally mitigate and prevent imminent Aedes-borne infections.

Additionally, due to the rising threat of global environmental and social change, which includes increased urbanization, trade, agricultural expansion, population growth, natural resource depletion, and climate change, further research and development on novel vector control methods is essential to anticipate resurgent or new arboviral infections. Furthermore, the potential for insecticide and gene drive resistance demonstrates that vector control methods, that were once considered to be effective, might need to be reconsidered or modified. Moreover, by coordinating research activities and sharing data within and between countries, research outcomes can be maximized, and data repetition avoided. For example, the Pan American Health Organization (PAHO) Health Information Platform is a real-time, voluntary, electronic reporting system that provides rapid access and shared data reporting within the Americas. Overall, cross-border collaborations and shared evidence-based research among countries have created numerous international guidelines, such as the Integrated Aedes Management (IAM) and the WHO Global Vector Control Response (GVCR) framework, which help health authorities implement effective, integrated, community-based, and locally adapted vector control strategies. In conclusion, using the existing vector control practices in place, combined with continued integrated vector management and global cooperation, can help combat the emerging threat of Aedes-borne viruses and improve human and environmental health and economies worldwide.

\section{References}

1. Aya Pastrana N, Lazo Porras M, Miranda JJ, Beran D, Suggs LS (2020) Social marketing interventions for the prevention and control of neglected tropical diseases: A systematic review. PLoS Neglected Tropical Diseases 14(6): e0008360.

2. Bonizzoni M, Gasperi G, Chen X, James AA (2013) The invasive mosquito species Aedes albopictus: Current knowledge and future perspectives. Trends Parasito, 29(9): 460-468.

3. Carvalho MS, Honorio NA, Garcia, LMT, de Sa Carvalho LC (2017) Aedes aegypti control in urban areas: A systemic approach to a complex dynamic. PLoS Neglected Tropical Diseases 11(7): e0005632.

4. Dusfour I, Vontas J, David JP, Weetman D, Fonseca DM (2019) Management of insecticide resistance in the major Aedes vectors of arboviruses: Advances and challenges. PLoS Neglected Tropical Diseases 13(10): e0007615.

5. (2017) European Centre for Disease Prevention and Control (ECDC) Vector control with a focus on Aedes aegypti and Aedes albopictus mosquitoes: Literature review and analysis. ECDC. 
6. Espinal MA, Andrus JK, Jauregui B, Waterman SH, Morens DM (2019) Emerging and reemerging Aedes-transmitted arbovirus infections in the region of the Americas: Implications for health policy. AJPH Perspectives from the Social Sciences 109(3): 387-392.

7. Houé V, Bonizzoni M, Failloux AB (2019) Endogenous non-retroviral elements in genomes of Aedes mosquitoes and vector competence. Emerging Microbes \& Infections 8(1): 542-555.

8. Huang B, Montgomery BL, Adamczyk R, Ehlers G, van den Hurk AF (2020) A LAMP-based colorimetric assay to expedite field surveillance of the invasive mosquito species Aedes aegypti and Aedes albopictus. PLoS Neglected Tropical Diseases 14(3): e0008130.

9. Kistler KE, Vosshall LB, Matthews BJ (2015) Genome engineering with CRISPR-Cas9 in the mosquito Aedes aegypti. Cell Reports 11(1): 51-60.

10. Kraemer M, Sinka ME, Duda KA, Mylne AQN, Shearer FM (2015) The global distribution of the arbovirus vectors Aedes aegypti and Ae. albopictus. eLife 4: e08347.

11. Macias VM, Ohm JR, Rasgon JL (2017) Gene drive for mosquito control: Where did it come from and where are we headed? International Journal of Environmental Research and Public Health 14(9): 1006.

12. Ortega López LD, Pondeville E, Kohl A, León R, Pazmiño Betancourth M (2020) The mosquito electrocuting trap as an exposure-free method for measuring human-biting rates by Aedes mosquito vectors. Parasites \& Vectors 13(1): 31.

13. Rochlin I, Ninivaggi DV, Hutchinson ML, Farajollahi A (2013) Climate Change and Range Expansion of the Asian Tiger Mosquito (Aedes

ISSN: 2574-1241

DOI: 10.26717/BJSTR.2021.34.005525

Satesh Bidaisee. Biomed J Sci \& Tech Res (C) This work is licensed under Creative

Submission Link: https://biomedres.us/submit-manuscript.php albopictus) in Northeastern USA: Implications for Public Health Practitioners. PLoS ONE 8(4): e60874.

14. Roiz D, Wilson AL, Scott TW, Fonseca DM, Jourdain F (2018) Integrated Aedes management for the control of Aedes-borne diseases. PLoS Neglected Tropical Diseases 12(12): e0006845.

15. Sánchez CHM, Bennet JB, Wu SL, Rasic G, Akbari OS (2020) Modeling confinement and reversibility of threshold-dependent gene drive systems in spatially-explicit Aedes aegypti populations. BMC Biology 18: 50 .

16. Scolari F, Casiraghi M, Bonizzoni M (2019) Aedes spp. and their microbiota: A review. Frontiers in Microbiology 10: 2036.

17. Sippy R, Rivera GE, Sanchez V, Heras F, Morejón B, Beltrán E, Neira M (2020) Ingested insecticide to control Aedes aegypti: Developing a novel dried attractive toxic sugar bait device for intra-domiciliary control. Parasites \& Vectors 13: 78

18. Wilson AL, Courtenay O, Kelly Hope LA, Scott TW, Takken W (2020) The importance of vector control for the control and elimination of vectorborne diseases. PLoS Neglected Tropical Diseases 14(1): e0007831.

19. Wooding M, Naudé Y, Rohwer E, Bouwer M (2020) Controlling mosquitoes with semio chemicals: A review. Parasites \& Vectors 13(1): 80.

20. (2017) World Health Organization (WHO) Global vector control response 2017-2030. Geneva: WHO. License: CC BY-NC-SA 3.0 IGO.

$\begin{array}{ll}\text { BIOMEDICAL } & \text { Assets of Publishing with us } \\ \text { RESEARCHES } & \text { - Global archiving of articles } \\ \text { - Immediate, unrestricted online access } & \text { - Rigorous Peer Review Process } \\ & \text { - Authors Retain Copyrights } \\ \end{array}$

